\title{
Análisis del movimiento en masa de la Quebrada Blanca: implicancias en la dinámica geomorfológica y criogénica. Cuenca superior del río Elqui
}

\author{
Pablo Rodrigo Iribarren Anacona \\ Unidad de Glaciología y Nieves. Dirección General de Aguas. Santiago, Chile. \\ pablo.iribarren@mop.gov.cl
}

\begin{abstract}
RESUMEN
Se estudia un movimiento en masa complejo ocurrido en la cuenca superior del Río Elqui, con el fin de evidenciar los factores desencadenantes e interpretar su relación con la dinámica geomorfológica, criogénica y climática actual. Mediante el análisis de imágenes satelitales, fotografías áreas y trabajo de campo se determinan algunos parámetros morfológicos del evento y se estima de manera aproximada la fecha de origen del fenómeno. El deslizamiento rotacional y el flujo detrítico asociado ocurrieron entre los años 1987-1988, afectaron un área de $0.22 \mathrm{~km}^{2}$ y movilizaron un volumen aproximado de materiales de 2.4-3.2 $\times 10^{6} \mathrm{~m}^{3}$. La sobreelevación de los depósitos (run up) de entre 15 y 50 metros permite estimar que el flujo detrítico habría alcanzado velocidades máximas de $31 \mathrm{~m} / \mathrm{s}$ en el contacto con los glaciares rocosos de la zona. Se advierte el importante rol que puede jugar el deshielo en años excepcionalmente nivosos en la génesis o evolución de los movimientos en masa y la utilidad de la información meteorológica, de los catálogos sísmicos y de las imágenes satelitales históricas en el análisis geomorfológico retrospectivo. Se destaca finalmente el rol de los glaciares rocosos como agentes reguladores del transporte de detritos en las áreas de alta montaña y la importancia que pueden tener fenómenos de gran magnitud y poca frecuencia, como el movimiento en masa analizado, en el suministro detrítico de estas formas.
\end{abstract}

Palabras claves: Río Elqui, movimiento en masa, piso periglacial, suministro detrítico, glaciar rocoso.

\section{Analysis of the mass movement of quebrada blanca: implicances in the geomorphological and cryogenic dynamic. Upper elqui river basin}

\begin{abstract}
A complex mass movement was studied in the upper Elqui River basin with the aim of identify the triggering factors and interpret they relation with the actual geomorphic, cryogenic and climatic dynamic. Throughout the analysis of satellite images, aerial photos and field work were calculated some geomorphological parameters of this event and was possible estimate the approximate age of this phenomenon. The rotational landslide and associated debris flow was occurred between the years 19871988 , affected an area of $0.22 \mathrm{~km}^{2}$ and mobilized an approximated material volume of $2.4-3.2 \times 10^{6} \mathrm{~m}^{3}$. The run up of deposits between 15 and 50 meters indicates that the debris flow would have reached maximum speeds of $31 \mathrm{~m} / \mathrm{s}$ in the contact with the rock glaciers of the zone. In this sense is outlined the important role that can play the thaw in snowy years in the genesis or evolution of the mass movements and the utility of the meteorological information, the seismic catalogues and the historical satellital images in geomorphological back-analysis. Finally it is outlined the role of rock glaciers as regulatory agents of the debris transport in high mountain areas and the importance of large magnitude and low frequency phenomena, like the mass movement analyzed, in the debris supply of these landforms.
\end{abstract}

Key words: Elqui River, mass movement, periglacial belt, debris supply, rock glacier. 


\section{INTRODUCCIÓN}

Los movimientos en masa son fenómenos comunes en diferentes contextos morfoclimáticos, especialmente en aquellas áreas de montaña afectadas recientemente por glaciaciones y deglaciaciones (BALLANTYNE 2002). El retroceso glaciar, el descongelamiento de suelos permanentemente congelados, la desaparición de campos de nieve perenne y cambios en los regímenes de fusión nival generan importantes alteraciones en las condiciones térmicas, hidráulicas y mecánicas del sustrato haciendo a estas áreas más propensas a generar desprendimientos u otro tipo de procesos gravitacionales (KÄ̈̈B et al. 2005).

Las investigaciones en distintas cadenas montañosas glaciadas del planeta han enfatizado el análisis de la relación existente entre el retroceso de los glaciares y la inestabilidad de laderas en las áreas de montaña (BALLANTYNE 2002). Además, han puesto énfasis en la caracterización de los movimientos en masa que han afectado glaciares blancos o descubiertos y la respuesta de estos glaciares frente a dichos fenómenos (DELINE 2008; HEWITT 2009; SCHULMEISTER et al. 2009). No obstante, son escasos los estudios que documentan la ocurrencia de grandes deslizamientos, desplomes o flujos de detritos que han afectado directa o indirectamente glaciares rocosos (KRYSIECKI et al 2008).

La génesis de los movimientos en masa varía caso a caso, sin embargo, en Los Andes centrales de Chile y Argentina parte importante de estos fenómenos han estado asociados a áreas con actividad sísmica poco profunda (ANTINAO y GHOSSE 2008), a años húmedos relacionados con el fenómeno de El Niño (MOREIRAS 2005), a altas tasas de fusión nival en la época estival (CASASSA y MARANGUNIC 1993) y a actividad volcánica (ABELE 1982). En la cuenca superior del Río Elqui no existen inventarios sistemáticos que documenten la existencia de movimientos en masa de gran magnitud $\left(>0,1 \mathrm{~km}^{2}\right)$ ocurridos en el pasado reciente. No obstante, existen evidencias geomorfológicas de movimientos en masa prehistóricos de grandes dimensiones como los que represaron el actual embalse La Laguna (ABELE 1984), la Laguna el Cepo y la avalancha de rocas del Cerro El Volcán (IRIBARREN 2008).

En el centro norte de Chile, a excepción del análisis del deslizamiento y posterior flujo

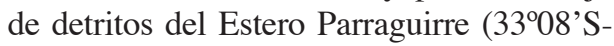
$70^{\circ} 01^{\prime} \mathrm{W}$ ) (CASASSA y MARANGUNIC 1993); HAUSER 1993; HAUSER 2002) y del análisis del colapso de un glaciar rocoso a los pies del Cerro Las Tórtolas (29 $58^{\circ}$ 'S$69^{\circ} 55^{\prime} \mathrm{W}$ ) (IRIBARREN y BODIN 2010) no existen estudios de movimientos en masa que hayan afectado en tiempos históricos zonas con permafrost de montaña, desconociéndose aún el comportamiento y verdadero alcance de este tipo de fenómenos.

En este contexto, se analiza el área afectada por un movimiento en masa histórico en la alta montaña de los Andes semiáridos de Chile, con el fin de identificar los posibles factores desencadenantes e interpretar su relación con la dinámica geomorfológica, criogénica y climática actual.

\section{MATERIALES Y MÉTODOS}

\section{Contexto geográfico}

El área de estudio se localiza entre los $30^{\circ} 12^{\prime}-30^{\circ} 13^{\prime} \mathrm{S}$ y $\operatorname{los} 69^{\circ} 55^{\prime}-69^{\circ} 57^{\prime} \mathrm{W}$ en la cuenca de la Quebrada Blanca, tributario del Río Colorado en la Región de Coquimbo. La cuenca de la Quebrada Blanca tiene una superficie aproximada de $6.4 \mathrm{~km}^{2}$ con elevaciones que fluctúan entre los 3650 y los 4950 m.s.n.m. Ésta tiene su desembocadura en las cercanías de la Ruta 41 que une la Región de Coquimbo con la Provincia de San Juan en Argentina (Fig.1).

La zona posee un clima de tundra de alta montaña, con gran sequedad atmosférica, 


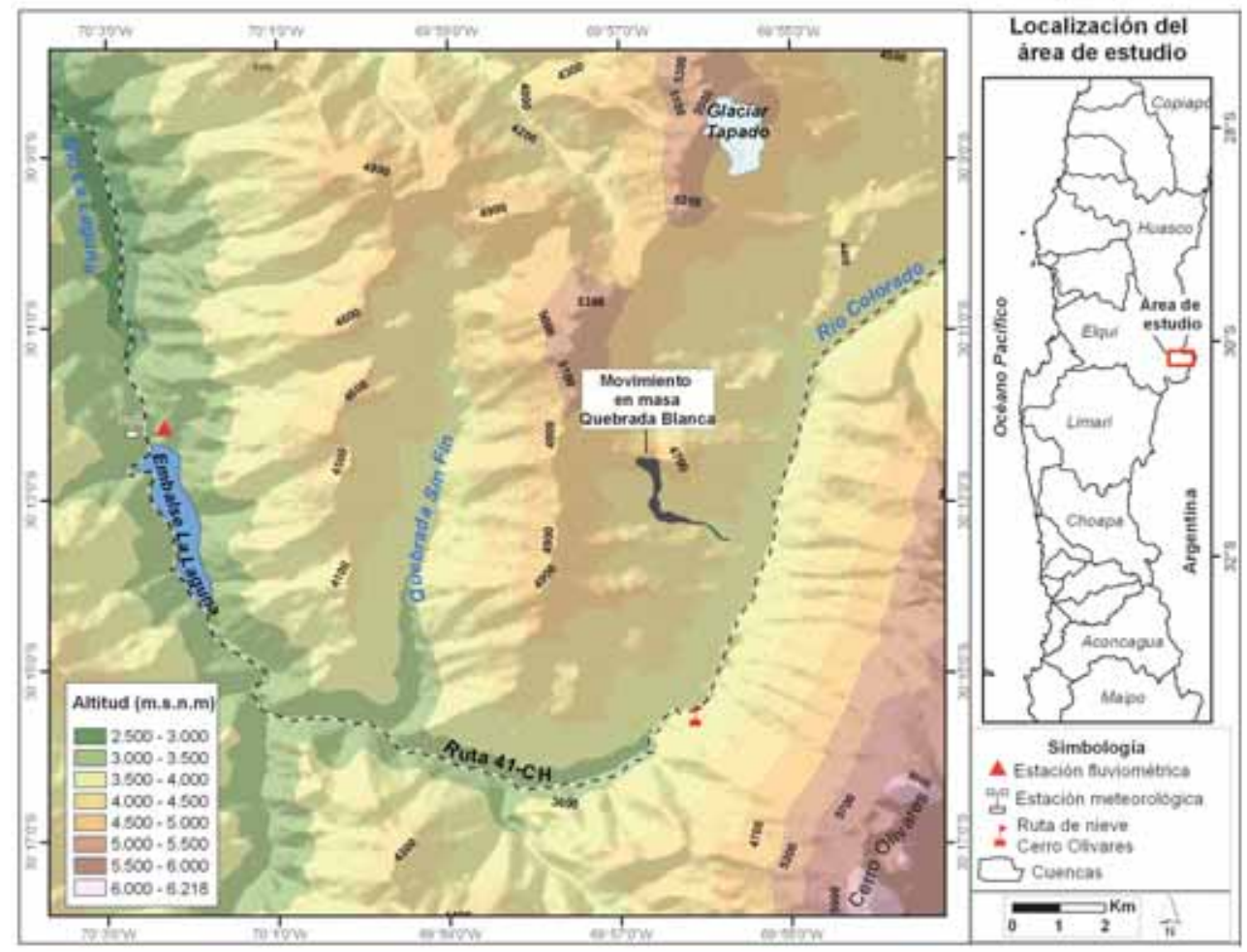

Fig.1

caracterizado por presentar veranos secos y cálidos, inviernos húmedos y una gran variabilidad interanual en las precipitaciones, condiciones directamente relacionadas con la variación estacional del Anticiclón del Pacífico Sur y con la intensidad de la manifestación de los fenómenos de El Niño y La Niña (ANTONIOLETTI et al. 1971; ACEITUNO 1988).

El paisaje criogénico de la región se caracteriza por presentar una gran cantidad de glaciares rocosos, localizados generalmente sobre los 3.700 m.s.n.m y por la reducida superficie ocupada por glaciares blancos o descubiertos. En la cuenca del Río Elqui existirían más de 200 glaciares rocosos (Dirección General de Aguas 2008) y 123 cuerpos de hielo descubierto, entre glaciares, glaciaretes y campos de nieve perenne. Estos últimos ocupan una superficie de 8,7 $\mathrm{km}^{2}$ (Dirección General de Aguas 2009) mientras que la superficie ocupada por gla- ciares rocosos entre los $29^{\circ}$ y los $30^{\circ}$ de latitud sur sería cercana a los $24 \mathrm{~km}^{2}$ (AZOCAR y BRENNING 2009).

La cuenca de la Quebrada Blanca posee una impronta glacial-periglacial reflejada en formas morrénicas, grandes conos de gravedad y numerosos glaciares rocosos. Geológicamente está constituida por rocas volcánicas estratificadas del Paleozoico Superior-Triásico Inferior y por un área con alteración hidrotermal (MPODOZIS y CORNEJO 1988).

\section{Procedimientos}

Las formas asociadas al movimiento en masa fueron identificadas y mapeadas mediante el análisis de una imagen multiespectral QuickBird (2.4 metros de resolución espacial), de marzo del año 2007, y mediante trabajo de campo realizado en marzo del año 2009. La nomenclatura geomorfológica 
utilizada para la descripción del fenómeno se basó en la propuesta de ÁLCANTARA (2000) adaptada de los criterios de VARNES (1978).

Se calculó la velocidad del movimiento en masa en dos secciones de la cuenca, considerando la sobre elevación de los depósitos que cubrieron parte de las laderas del valle y glaciares rocosos de la zona. Ésto se realizó mediante la fórmula de sobre elevación:

$$
\mathrm{V}=(2 \mathrm{gh})^{0.5}
$$

Donde V es la velocidad del flujo, g es la aceleración de gravedad, y h es la altura de sobre elevación de los depósitos, medida perpendicularmente al flujo. Esta fórmula, propuesta por CHOW (1959), no considera la fricción interna y basal del flujo. No obstante, los experimentos realizados muestran una subestimación de hasta un $30 \%$ de su velocidad real (IVERSON et al. 1994).

Debido a la inexistencia de investigaciones que documentarán la fecha en que ocurrió este fenómeno y a los rasgos morfológicos que hacen presumir su reciente data, se realizó una revisión de las fotografías aéreas disponibles de la zona e imágenes satelitales históricas para hacer una aproximación a su fecha de origen.

Para ello se analizaron las fotografías aéreas $\mathrm{N}^{\circ} 16134$ del vuelo Geotec del año 2000 y la fotografía $\mathrm{N}^{\circ} 26140$ del vuelo Hycon del año 1956 a escala 1:50.000. Posteriormente se revisaron imágenes satelitales Landsat de las décadas de los ochenta y noventa de los sensores Thematic Mapper y Enhanced Thematic Mapper Path/Row 233/81 disponibles en el sitio web del United Stated Geological Survey (http//Landsat.usgs. gov/). Estas imágenes multiespectrales, de 30 metros de resolución espacial, fueron comparadas visualmente en la combinación falso color 5,4 y 2, desplegadas en los canales rojo, verde y azul, para detectar la presencia del movimiento en masa.
Los factores condicionantes y los posibles factores desencadenantes del movimiento en masa, fueron analizados poniendo énfasis en el periodo en que se habría desencadenado el evento. La información geológica fue extraída de MPODOZIS y CORNEJO (1988), la información sísmica fue extraída del catálogo sísmico del International Seismological Center (2009), y la información meteorológica de los registros de la estación La Laguna perteneciente a la Dirección General de Aguas. Los años con ausencia de registros de precipitación fueron reconstruidos mediante regresión lineal utilizando los datos de la estación meteorológica Rivadavia (820 m.s.n.m; $29^{\circ} 58^{\prime}$ 'S-70³3'W).

Para analizar la posible relación existente entre la actividad sísmica y el desencadenamiento del fenómeno, se calculó la aceleración producida por los sismos con epicentros cercanos al área de estudio y por aquellos eventos de mayor magnitud registrados en la región utilizando la ley de atenuación de Intensidad Arias de WILSON y KEEFER (1985):

$$
\begin{aligned}
& \log 10 I a=-4.1+\mathrm{M}-2 \log 10\left(\mathrm{~d}^{2}+\mathrm{h}^{2}\right)^{0.5} \\
& -0.5 \mathrm{P}
\end{aligned}
$$

Donde Ia corresponde a la Intensidad Arias en $\mathrm{m} / \mathrm{s}, \mathrm{M}$ a la magnitud del sismo (aquí se utilizaron datos de Ms), d a la distancia desde la zona de origen del deslizamiento hasta la proyección de ruptura en $\mathrm{km}, \mathrm{h}$ a la profundidad del hipocentro en $\mathrm{km}$, y $\mathrm{P}$ a un término de probabilidad que incluye posibles variaciones e incertidumbre, aquí se consideró como 0 en todos los casos. Se tomaron como referencia de $I a$ los umbrales establecidos por HARP y WILSON (1995), que indican que movimientos en masa como deslizamientos o asentamientos profundos, se generan con $I a$ superiores a $0.32 \mathrm{~m} / \mathrm{s}$.

Cabe señalar, que ésta es sólo una aproximación general, ya que se necesitan datos geotécnicos como la fricción interna, densidad y grado de saturación de los materiales en el momento que ocurrió el movimiento 
en masa, para determinar efectivamente si un evento sísmico pudo ser el desencadenante del fenómeno.

\section{RESULTADOS}

\section{Descripción del movimiento en masa}

La zona más elevada del movimiento en masa se ubica entre los 4.600 y los 4.700 m.s.n.m y está constituida por rocas volcánicas pertenecientes a la Formación Pastos Blancos (Fig.2.). La ladera afectada tiene una exposición sureste con una pendiente promedio original de $32^{\circ}$. La superficie de ruptura tiene un área planimétrica de $0.08 \times 10^{6} \mathrm{~m}^{2}$ y la altura media del escarpe principal es de 30-40 metros, con ello se estima un volumen de material movilizado de 2.4-3.2 $\times 10^{6} \mathrm{~m}^{3}$ el que probablemente se incrementó con materiales incorporados desde el talweg que presenta rasgos erosivos. La zona de origen presenta numerosas grietas de tensión y en el sector noroccidental, escarpes pronunciados y cuerpos secundarios hundidos en una superficie cóncava que permiten evidenciar la existencia de deslizamientos rotacionales sincrónicos o posteriores al evento principal (Fig. $3 a$ y $3 b$ ).
El cuerpo principal del movimiento en masa, sin embargo, se transformó en un flujo detrítico que cubrió una superficie de alrededor de $0.22 \mathrm{~km}^{2}$ con un alcance de un poco más de $2 \mathrm{~km}$. El flujo se encauzó por un tributario de la Quebrada Blanca en dirección sur y se proyectó contra la vertiente sur del valle cubriendo la parte frontal del glaciar rocoso 1 (Fig.4). Desde este punto presenta un cambio de dirección en cerca de $90^{\circ}$ orientándose hacia el este, donde cubrió el fondo del valle y el frente de los glaciares rocosos 5 y 6 . La altura de sobre elevación de los depósitos (entre 15 y 50 metros) indica que el flujo podría haber alcanzado velocidades máximas de entre 17 y $31 \mathrm{~m} / \mathrm{s}$ en la zona de contacto con los glaciares rocosos (Fig. 5a y 5b).

El análisis de las imágenes satelitales y fotografías aéreas disponibles da cuenta de que el deslizamiento y posterior flujo detrítico se produjo entre el 30/01/1987 y el 13/08/1988. No obstante, la fotografía aérea del año 1956 ya mostraba evidencias de inestabilidad (pequeñas grietas de distensión) en la zona media de la ladera posteriormente afectada por el deslizamiento. En efecto, a pesar de que el área más elevada del movimiento en masa se ubica a pocas

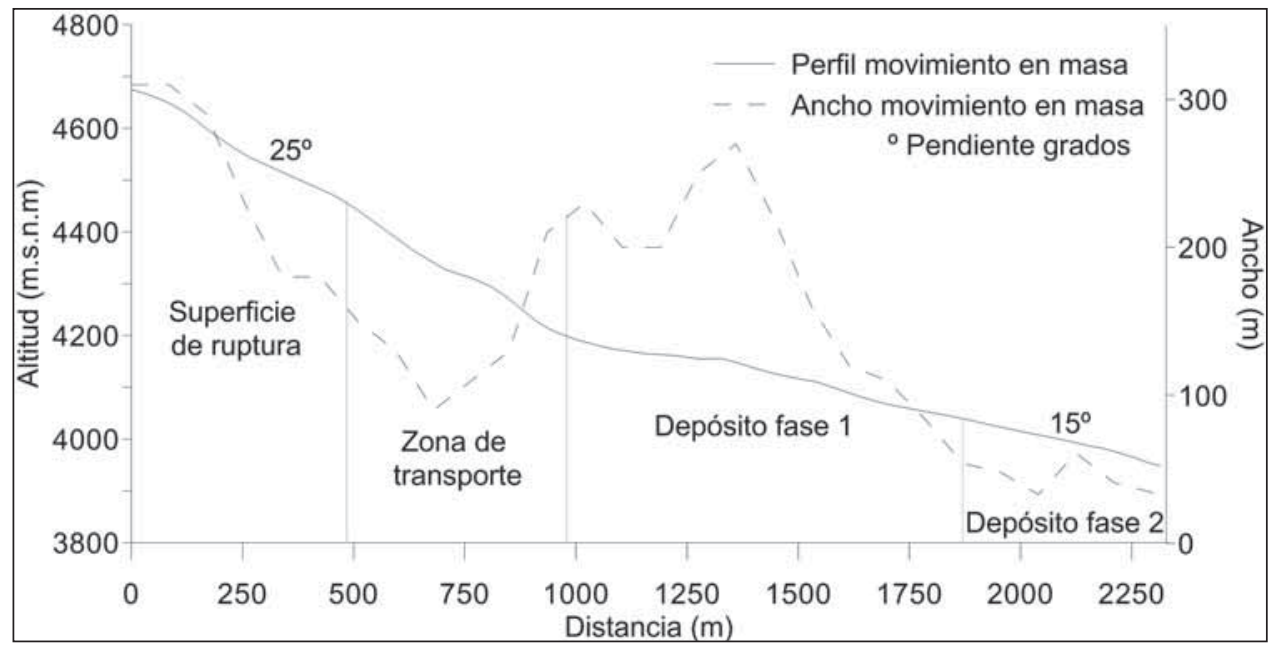

Fig.2 


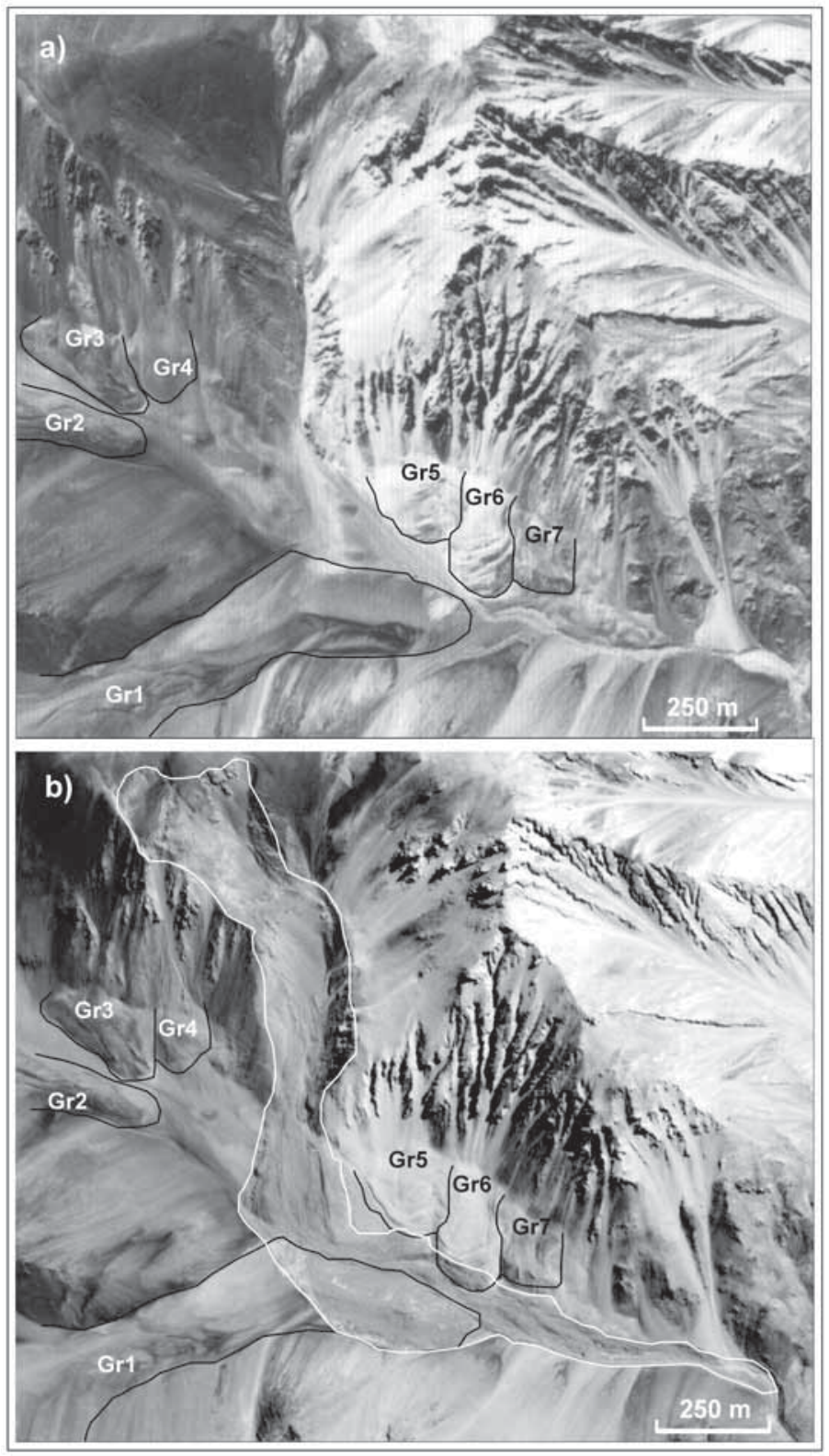

Fig.3

decenas de metros de la divisoria de aguas, la mayor parte del material movilizado proviene de su parte media.

\section{Características del depósito}

La morfología del depósito da cuenta de la existencia de distintas fases del movimiento en masa. Una de las fases se desa- rrolló en un medio más viscoso y dio origen al depósito de mayor potencia dentro de la quebrada de aproximadamente 10 metros de espesor (Fig. 5c). Este depósito posee forma lobulada, costados abruptos y pequeños cordones y surcos en su superficie producto de un flujo compresivo en el contacto con los glaciares rocosos 5 y 6 . 


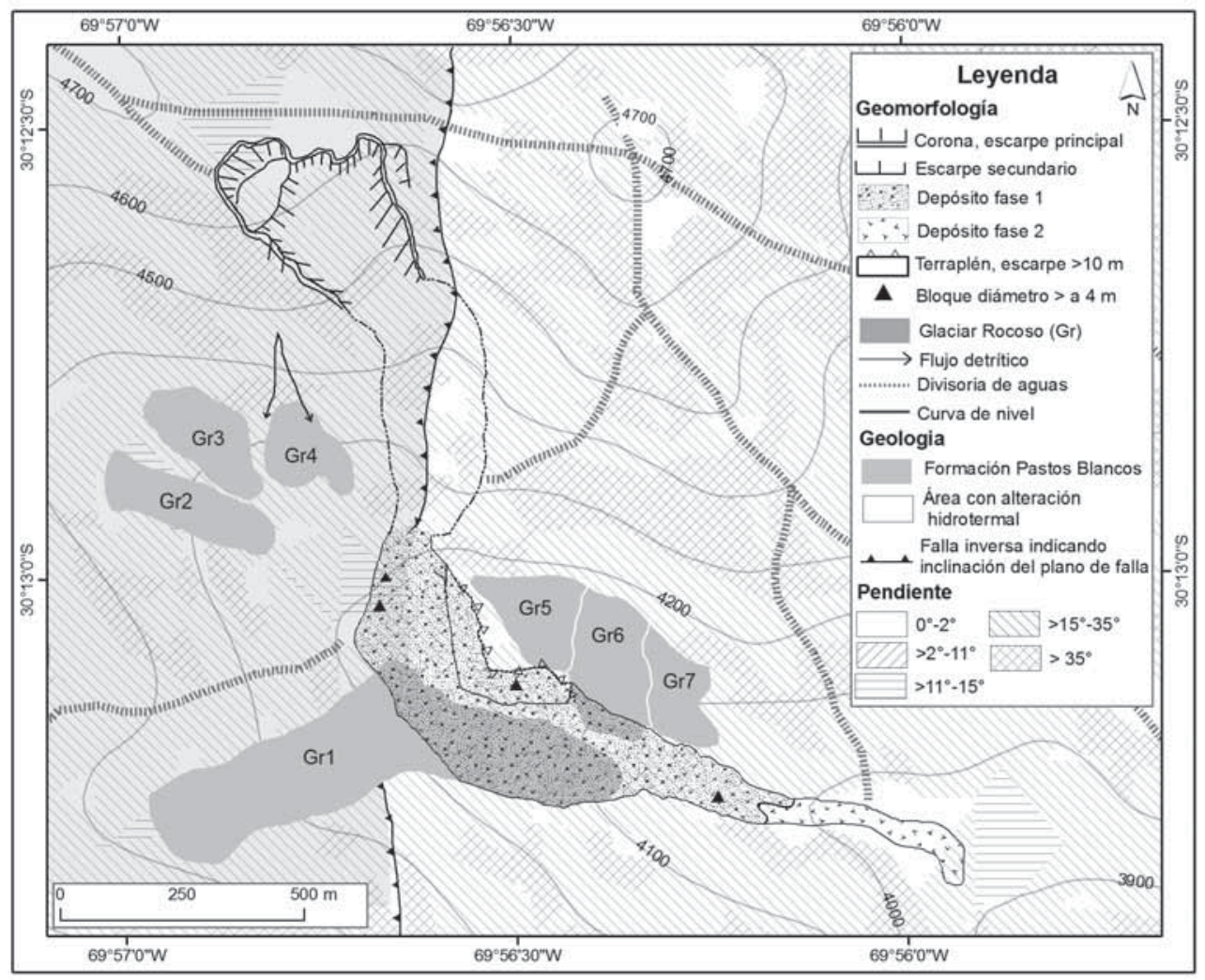

Fig.4

Las trazas de sobre elevación observadas a lo largo de la quebrada y los depósitos en forma laminar en el fondo del valle dan cuenta de otra fase del movimiento en masa, de menor potencia, que probablemente se desarrolló en un medio más fluido (Fig.6). Esta fase tiene su mayor expresión en los últimos $500 \mathrm{~m}$ del depósito aunque es observable también sobre los glaciares rocosos antes mencionados.

El depósito está compuesto principalmente de gravas y bloques subangulares con matriz arenosa. Los bloques de mayores dimensiones tienen $4 \mathrm{~m}$ de diámetro y se ubican en el extremo occidental del terraplén desarrollado en el contacto entre el talweg desde donde provino el movimiento en masa y el valle de la Quebrada Blanca (Fig.7). Cabe destacar, que los bloques de mayor tamaño presentan un alto grado de descomposición, siendo en muchos casos disgregables por presión manual.

La descomposición de los bloques ha generado una topografía acolinada caracterizada por la presencia de conos pardo rojizos y ocres que varían desde unas pocas decenas de centímetros hasta un par de metros de alto (Fig.7). Dichas formas se encuentran incorporadas en el depósito y también proyectadas varios metros fuera de este. La morfología y heterogeneidad de las rocas que componen los conos hacen presumir que algunas de estas formas, específicamente las de menor diámetro y altura, podrían corresponder también a conos sucios o cubiertos. Estas formas se originan por la fusión diferencial de parches de nieve o cuerpos de hielo cubiertos por detritos. Cabe señalar, sin embargo, que se analizó el interior de algunas de estas formas sin encontrar nieve o hielo en su interior. 

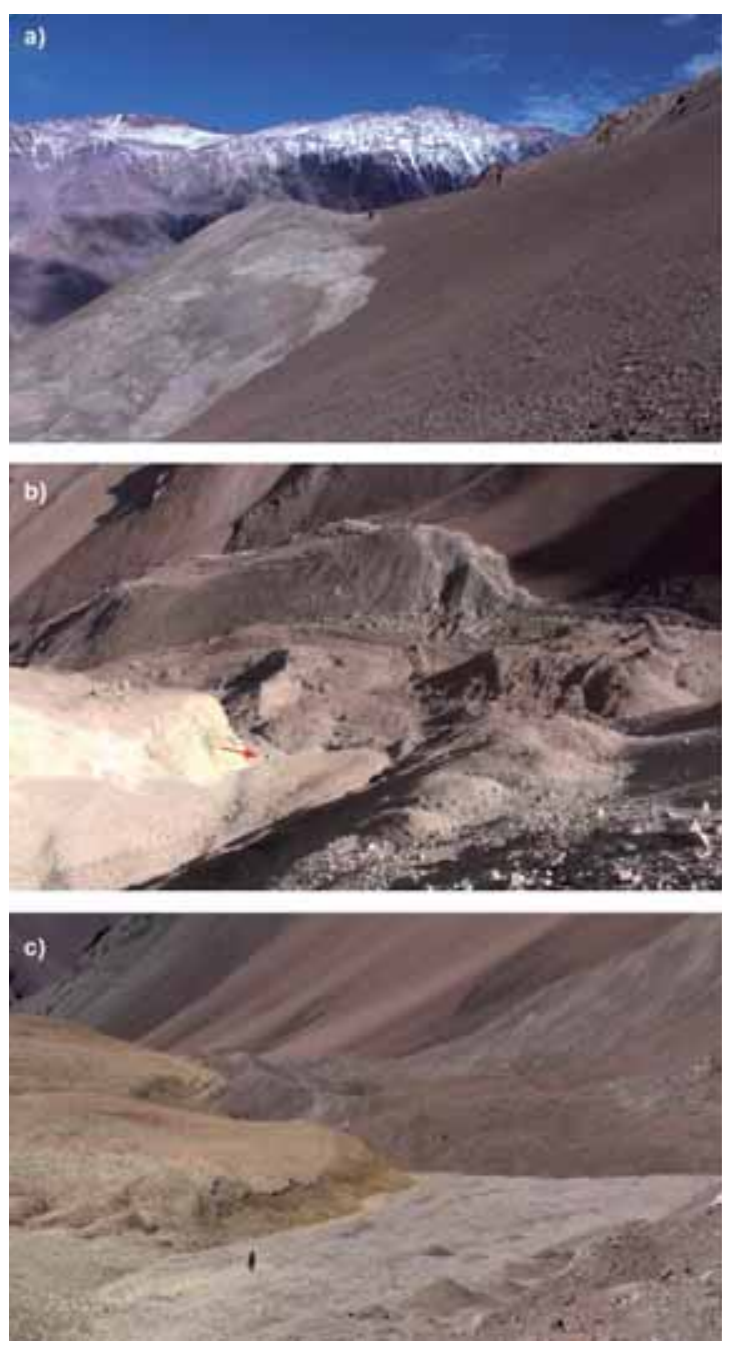

Fig.5

\section{Mecanismo del movimiento en masa}

\section{Factor geológico}

El área de origen del deslizamiento y posterior flujo detrítico se encuentra en la zona de contacto entre las riolitas, brechas volcánicas, lavas y tobas de la formación Pastos Blancos y un área con alteración hidrotermal. El contacto entre estas unidades se produce a través de una falla inversa de rumbo norte-sur que habría estado activa en el límite del Mioceno Inferior-Mioceno Medio (MPODOZIS y CORNEJO 1988). La debilidad estructural de la ladera y el alto grado de alteración de las rocas a causa del fallamiento, sumados a una pendiente superior a $\operatorname{los} 30^{\circ}$, hacen a la zona altamente susceptible a generar movimientos en masa y constituyen, probablemente, el factor condicionante que en mayor medida explica la ocurrencia del fenómeno. 


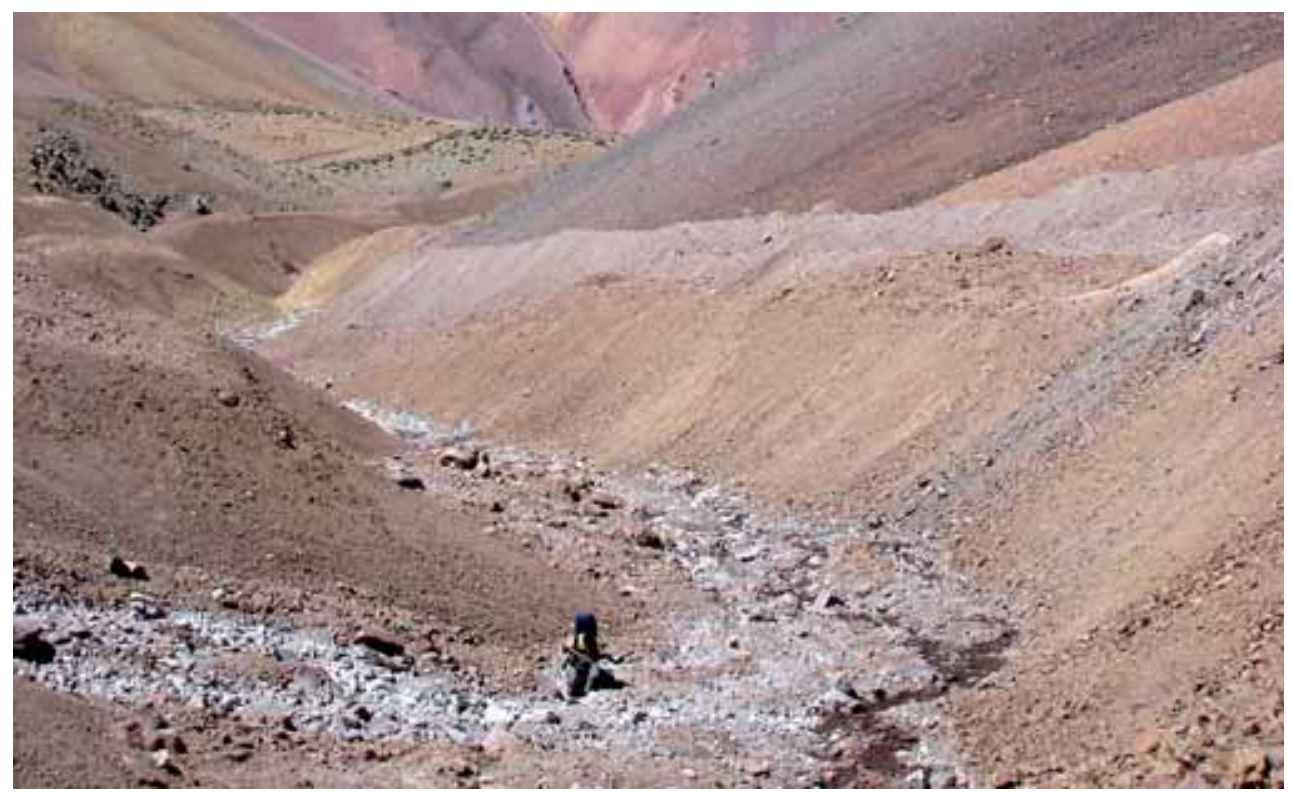

Fig.6

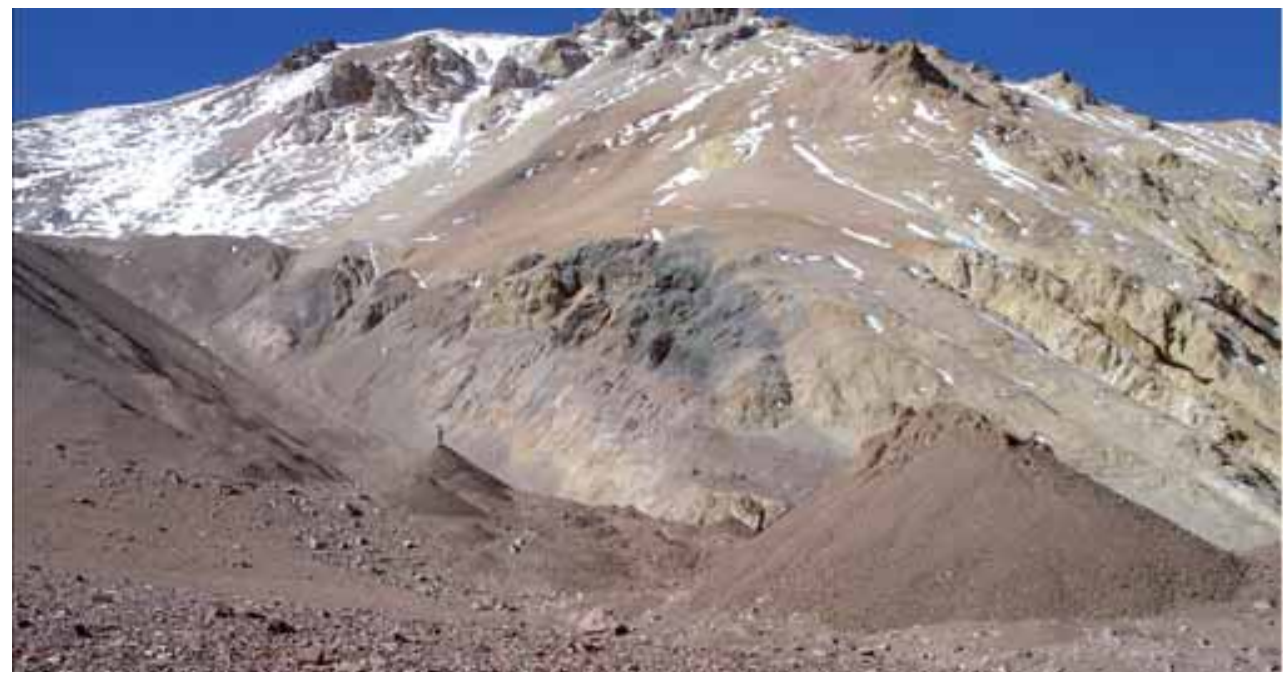

Fig.7

\section{Actividad sísmica}

En la Fig. 8 se ilustra la localización y la magnitud de los sismos registrados en la región cercana al área de estudio entre el $30 / 01 / 1987$ y el 13/08/1988. La actividad sísmica en este periodo estuvo concentrada en el sector costero siendo el evento de mayor magnitud un sismo de $6 \mathrm{Ms}$. El sismo ocurrió el 19 de mayo de 1987 a unos 150 $\mathrm{km}$ de la Quebrada Blanca, en las cercanías de la localidad de Tongoy, fue percibido con una intensidad V en la escala de Mercalli en Vicuña, y tuvo su hipocentro a $44 \mathrm{~km}$ de profundidad.

En la figura 8 se observa además que los sismos con focos en el cordón andino fueron escasos. Los dos eventos más cercanos al deslizamiento se ubicaron a distancias de 20 y $24 \mathrm{~km}$ de su zona de origen, tuvieron magnitudes de $4.1 \mathrm{Ms}$, profundidades de 
104 y $186 \mathrm{~km}$ y ocurrieron en marzo y julio del año 1988 respectivamente. Estos corresponden a sismos intraplaca de profundidad intermedia.

Con estos datos se calculó la Intensidad Arias producida en la zona de origen del movimiento en masa por dichos sismos y por el evento de mayor magnitud registrado en el periodo analizado. Los resultados en todos los casos fueron inferiores a $0.32 \mathrm{~m} / \mathrm{s}$ en varios orde- nes de magnitud. Este umbral es considerado crítico para provocar un movimiento en masa de las características del fenómeno estudiado (HARP y WILSON 1995).

\section{Factor meteorológico}

Los registros de precipitación de la estación meteorológica La Laguna (3.160 m.s.n.m), ubicada a unos $10 \mathrm{~km}$ al oeste del área de estudio, dan cuenta de precipitaciones

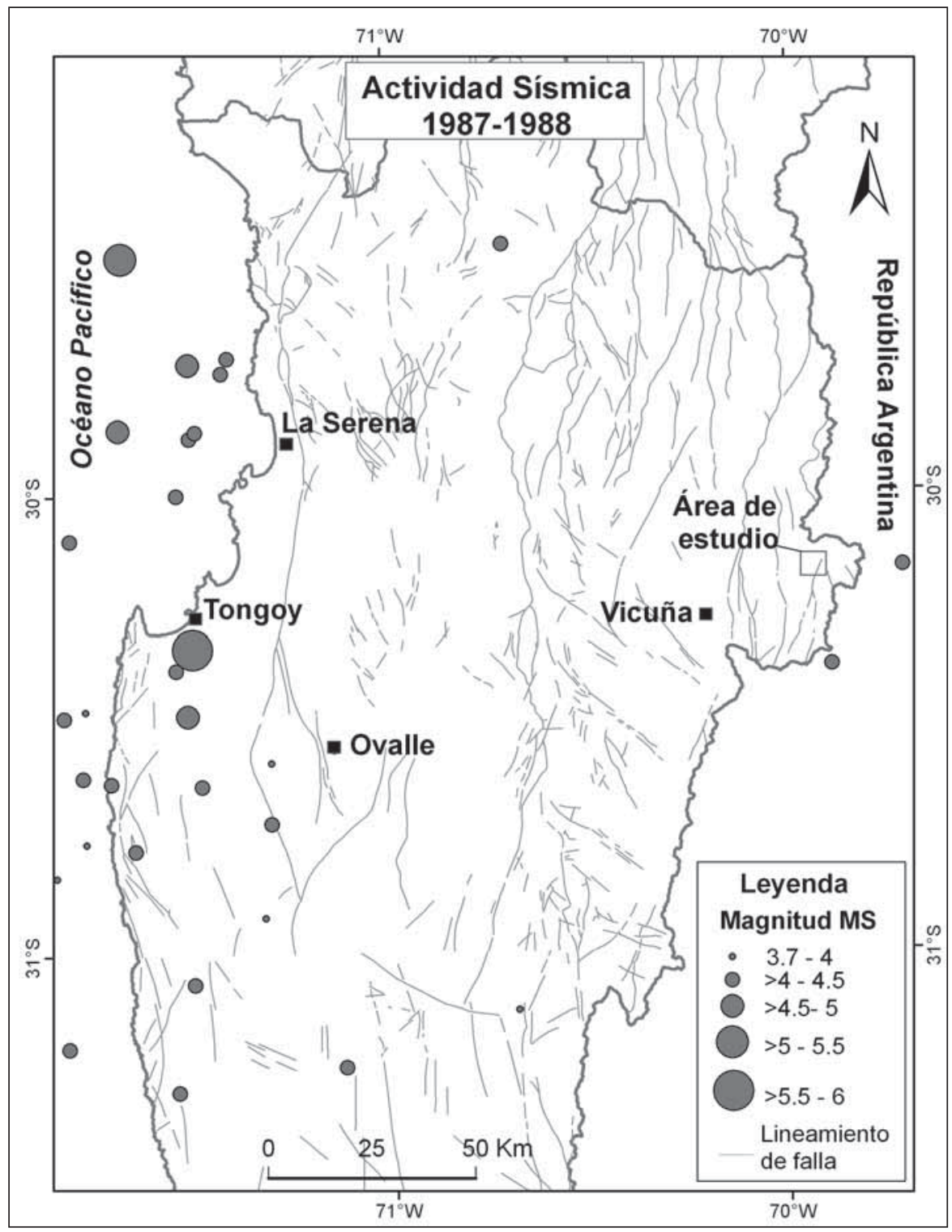

Fig. 8 


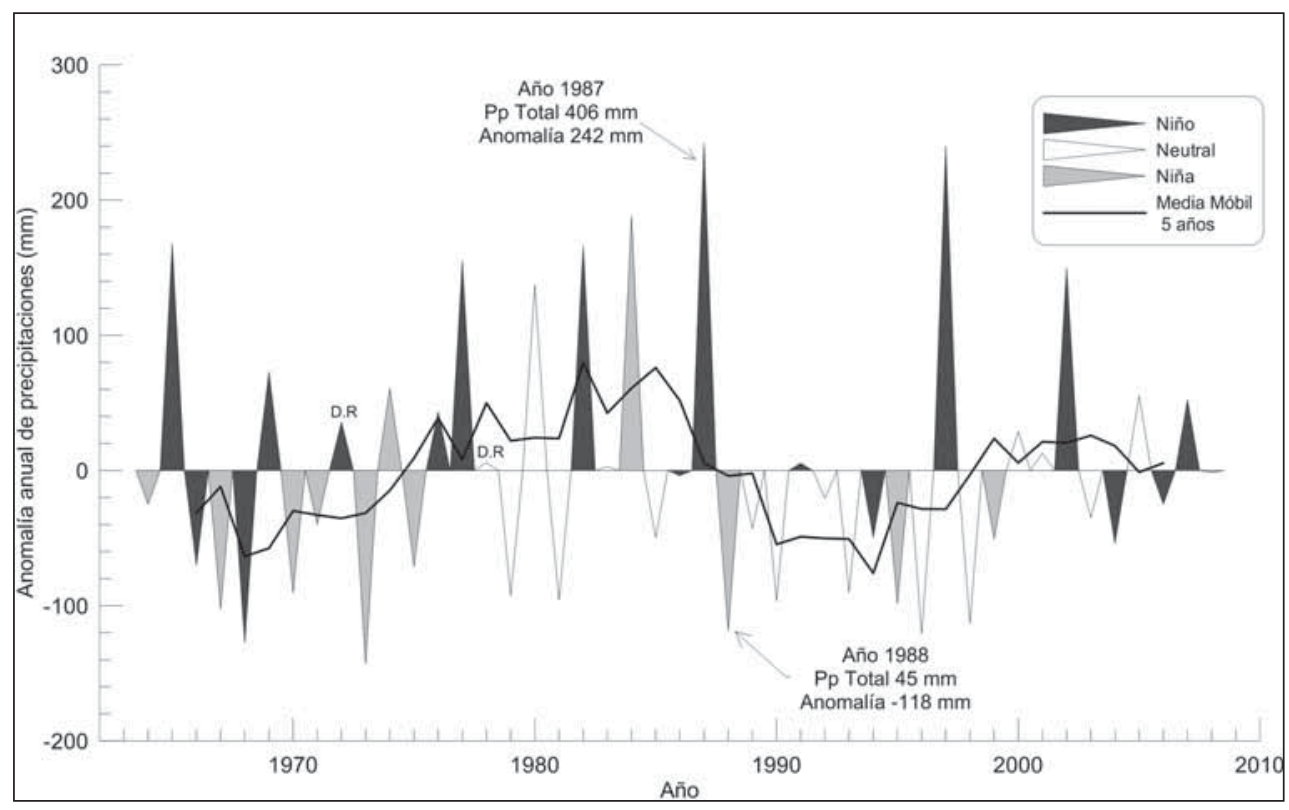

Fig. 9

medias anuales de $164 \mathrm{~mm}$ en el periodo 1964-2008. No obstante, en la figura 9 se observa que existe una significativa desviación del total anual de precipitaciones respecto a la media histórica.

Dicha variabilidad queda reflejada en el bienio 1987-1988, en el transcurso del cual ocurrió el movimiento en masa. El año 1987 fue el año que presentó el mayor monto de precipitaciones en el periodo 1964-2008, registrando un total de $406 \mathrm{~mm}$, monto 2.5 veces superior al promedio anual. El año 1988 en cambio, sólo precipitaron $45 \mathrm{~mm}$, siendo este el tercer año con menor cantidad de precipitaciones en 44 años de registro.

Los caudales medios mensuales del Río La Laguna reflejan un intenso periodo de deshielo en la primavera de 1987 y el verano de 1988, registrándose los caudales máximos en el mes de diciembre de 1987 (Fig.10). Considerando que la isoterma anual de cero grados centígrados en la cuenca se encuentra sobre los 4200 m.s.n.m, se estima poco probable que precipitaciones líquidas se registraran en la zona de origen del movi- miento en masa acelerando de esa manera el deshielo.

La intensa fusión nival también se evidencia al analizar los datos de la ruta de nieves del Cerro Olivares (3.550 m.s.n.m). El 4 de septiembre de 1987 se registró una profundidad de nieve de $1.12 \mathrm{~m}$ disminuyendo a 0.23 metros a mediados de noviembre. Ese mes registró una temperatura máxima de $25^{\circ} \mathrm{C}$, la quinta temperatura más alta registrada en noviembre desde 1964.

\section{DISCUSIÓN}

\section{Efecto de la sismicidad}

En ANTINAO y GOSSE (2008) se señala que en Chile Central la mayor parte de los deslizamientos rocosos están asociados a áreas con sismicidad superficial $(\leq 20 \mathrm{~km})$. De hecho en ERIKSON y HÖGSTEDT (2004) se advierte que a sismos interplacas con magnitudes de entre 7 y 8 Ms como los de Valparaíso (1906), Illapel (1943) y San Antonio-Valparaíso (1985) sólo han estado 


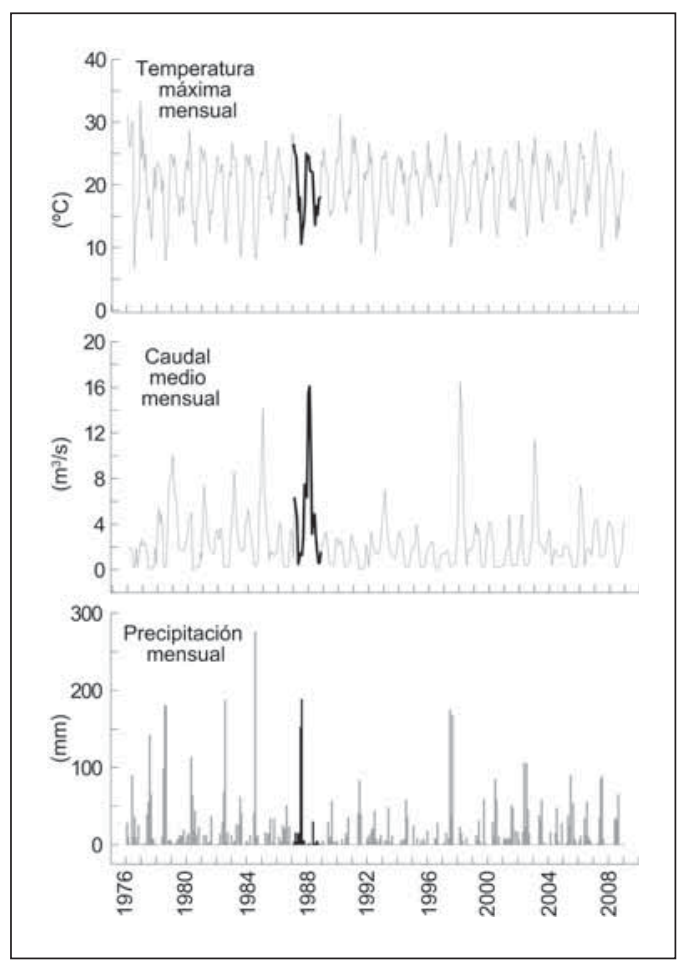

Fig. 10

asociados a pequeños desprendimientos de roca y deslizamientos detríticos.

Teniendo en cuenta estos antecedentes, los valores de Intensidad Arias, y aún considerando la probable amplificación topográfica de las ondas sísmicas en la zona de origen del deslizamiento, se estima poco probable que uno de los eventos sísmicos analizados haya sido el generador del movimiento en masa. No obstante, no se descarta que posibles eventos sísmicos de profundidad superficial no detectados por la red sismológica (ver PARDO et al. 2004) hayan tenido alguna influencia en la génesis del fenómeno.

\section{Factor meteorológico}

Las figuras 9 y 10 dejan de manifiesto el corto e intenso periodo de deshielo ocurrido en la región en la primavera del año 1987 y el verano de 1988 y con ello la incorporación sostenida de un importante volumen de agua en el sustrato. La potente acumulación nival asociada a una intensa manifestación del fenómeno de El Niño el año 1987, sumada a las altas tasas de fusión en primavera habría generado condiciones propicias para el desencadenamiento de movimientos en masa. Condiciones meteorológicas similares habrían estado asociadas a la avalancha de rocas y posterior flujo detrítico del Estero Parraguirre ocurrido en noviembre del mismo año en la cuenca del Río Colorado en Chile Central (CASASSA y MARANGUNIC 1993).

Investigaciones realizadas en otras cadenas montañosas del planeta han demostrado que en años con precipitaciones níveas excepcionalmente altas, la probabilidad de que ocurran movimientos en masa generados por fusión nival aumenta de manera considerable (KAWAGOE et al. 2009). La fusión nival puede elevar el nivel freático en materiales fuertemente fracturados y aumentar la presión de agua en los poros provocan- 
do su desestabilización y posterior colapso (MATHEWSON et al. 1990).

Aspectos morfológicos del depósito como la presencia de una delgada lámina de sedimentos $(\leq 40 \mathrm{~cm})$ que alcanzó gran proyección dentro de la quebrada, la presencia de cordones y surcos característicos de flujos compresivos en medios viscosos y la presencia de colinas y subsidencias locales derivadas probablemente de la fusión diferencial de parches de nieve cubiertos con detritos, confirman la presencia de agua o nieve en la masa detrítica movilizada. Estos elementos permiten corroborar la influencia al menos indirecta de las condiciones meteorológicas en la génesis o evolución del fenómeno.

\section{Impacto en el sistema criogénico}

A pesar de la gran velocidad que parece haber adquirido el flujo detrítico que impactó frontalmente al glaciar rocoso 1 (más de 100 $\mathrm{km} / \mathrm{hora}$ ) y a que los depósitos obstruyen el avance del glaciar rocoso 5 no se observaron evidencias de incorporación de un volumen importante de materiales de los glaciares al flujo ni una deformación significativa de estos cuerpos de hielo. Al cabo de 20 años de ocurrido el evento, los glaciares rocosos afectados por el flujo detrítico no han mostrado grandes cambios morfológicos.

Ésto puede deberse a la reducida potencia de los depósitos que cubrieron los glaciares ( $\leq 2$ metros) y a que estos materiales impactaron y cubrieron sólo parcialmente sus zonas frontales, las que habrían absorbido la mayor parte de la energía cinética del fenómeno.

Cabe señalar que los efectos que pueden tener los movimientos en masa de gran magnitud en ambientes criogénicos, varían notablemente según el tipo formas que afectan en su trayectoria. En la superficie de glaciares blancos o descubiertos, el alcance de los movimientos en masa puede incrementarse notablemente, no obstante, al cabo de pocas dé- cadas gran parte del material es redistribuido hacia los márgenes del glaciar o es incorporado al interior de los mismos a través de las grietas (HEWITT 2009). Los materiales incorporados en glaciares rocosos en cambio pueden permanecer varios miles de años en su superficie al ser integrados en un sistema de reptación lenta y posiblemente sólo son transferidos valle abajo en el transcurso de las glaciaciones (HUMLUM 2000).

Asumiendo que la tasa de retroceso de las vertientes en la región es $\leq 1 \mathrm{~mm} /$ año (SCHROTT 1996; BRENNING, 2005), que un área de $0.06 \mathrm{~km}^{2}$ del glaciar rocoso 1 fue cubierta por una capa de detritos de aproximadamente 2 metros de espesor y que el área abastecedora de detritos de este glaciar es de alrededor de $1.2 \mathrm{~km}^{2}$, se puede estimar que el deslizamiento y posterior flujo detrítico incrementaron el volumen de materiales que se incorpora anualmente a la superficie de este glaciar, en cerca de 2 órdenes de magnitud, el año en que ocurrió el evento.

Este hecho demuestra la importancia que pueden tener fenómenos de gran magnitud y poca frecuencia, como el movimiento en masa analizado, en el sistema de transportes de detritos en la alta montaña, en la alimentación de los glaciares rocosos y ulteriormente en la dinámica de estas formas.

\section{CONCLUSIONES}

Si bien no fue posible establecer con certeza el factor desencadenante del movimiento en masa, los rasgos morfológicos de los depósitos y el análisis de la actividad sísmica y de las condiciones meteorológicas de la región, hacen presumir fundadamente que éste podría estar asociado a las precipitaciones excepcionalmente altas ocurridas el año 1987 y al corto e intenso periodo de deshielo. Estos elementos, sumados a rocas poco resistentes y a una fuerte pendiente, habrían generado condiciones propicias para el desencadenamiento del movimiento en masa. Se destaca en este sentido la utilidad de las imágenes 
satelitales históricas, los catálogos sísmicos y la información meteorológica en el análisis geomorfológico retrospectivo.

Las observaciones realizadas en los glaciares rocosos afectados por el movimiento en masa permiten evidenciar el rol regulador del transporte de detritos de estas formas en las áreas de alta montaña. De hecho los glaciares rocosos no han sufrido cambios significativos desde que ocurrió el fenómeno hace más de dos décadas, permaneciendo los detritos incorporados por el flujo aún en su superficie.

Finalmente se destaca la importancia que puede tener el deshielo, especialmente en años excepcionalmente nivosos, en el desencadenamiento de movimientos en masa en la alta montaña del Río Elqui, en donde factores como la sismicidad superficial, asociada estrechamente a movimientos en masa en Chile Central (ANTINAO y GOSSE 2008) son menos recurrentes. Adquiere relevancia en este sentido la evaluación de los procesos geomorfológicos que puedan desencadenarse en la media y alta montaña de la región semiárida de Chile debido a factores meteorológicos, considerando que las modelaciones climáticas indican un probable aumento de las precipitaciones y un incremento de la temperatura en la región durante el presente siglo (DEPARTAMENTO DE GEOFÍSICA UNIVERSIDAD DE CHILE 2006).

Un aumento de la temperatura podría implicar la caída de precipitaciones líquidas en cotas más altas que las actuales en zonas donde existe una gran cantidad de detritos poco cohesionados o laderas inestables en cuencas susceptibles de generar flujos detríticos (e.g Quebrada el Calvario 29 $57^{\prime}$ S- $70^{\circ} 11^{\prime} \mathrm{W}$, ver PASKOFF 1993). Además, el aumento de la temperatura y los consiguientes cambios en los regímenes de deshielo podrían producir la desestabilización de las áreas con permafrost. Elementos importantes de considerar en la planificación territorial de las cuencas de alta montaña sobre todo si se tiene en cuenta la presencia de una ruta internacional, actividades turísticas y emprendimientos mi- neros enmarcados en el cordón andino de la Región de Coquimbo.

\section{AGRADECIMIENTOS}

El autor expresa su agradecimiento a Hales López y Cristian Anacona por su colaboración en el trabajo de campo. A Graciela Salinas de Salmuni de la Comisión Nacional de Actividades Espaciales de Argentina (CONAE), por facilitar el acceso a algunas de las imágenes satelitales utilizadas en el análisis, a Alexander Brenning por la revisión crítica de la versión preliminar de este trabajo y a José Araya, María Mardones y los editores de la revista por sus valiosas sugerencias.

\section{REFERENCIAS}

ABELE, G., 1984. Derrumbes de montaña y morrenas en los Andes chilenos. Revista de Geografía Norte Grande, 11:17-30.

ACEITUNO, P., 1988. On the functioning of the southern oscillation in the South American sector. I. Surface climate. Monthly Weather Review.116: 505-524.

ALCÁNTARA, I., 2000. Landslides: deslizamientos o movimientos del terreno? Definición, clasificaciones y terminología. Investigaciones Geográficas, Boletín, 41, Instituto de Geografía, UNAM, México. 7-25.

ANTINAO, J. y J. GOSSE., 2008. Large rockslides in the Southern Central Andes of Chile (32-34.5 $\mathrm{S})$ : Tectonic control and significance for Quaternary landscape evolution, Geomorphology.Doi10.10106/j.geomorph.2008.08.008

ANTONIOLETTI, R. J, BORCOSQUE. H, SHNEIDER., 1971. Características climáticas del norte chico IREN. P 102.

AZOCAR, G. y A. BRENNING., 2009. Hydrological and geomorphological sig- 
nificance of rock glaciers in the dry Andes, Chile $\left(27^{\circ}-33^{\circ} \mathrm{S}\right)$.Permafrost and periglacial processes. 21(1)42-53.

BALLANTYNE, C.K., 2002. Paraglacial geomorphology. Quat. Sci. R.,21:255-269

BRENNING, A., 2005. Climatic and geomorphological controls of rock glaciers in the Andes of Central Chile: Combining statistical modelling and field mapping. PhD dissertation, Humboldt-Universitat zu Berlin. urn:nbn:de:kobv:11-10049648.

CASASSA, G. y C. MARANGUNIC., 1993. The Río Colorado rockslide and debris flow, Central Andes, Chile: Bulletin of Association of Engineering Geologists, 30: 321-330.

CHOW,V., 1959. Open-channel hydraulics. Mcgrawl-Hill. New York, NY.P.680.

DELINE, P., 2008. Interactions between rock avalanches and glaciers in the Mont Blanc massif during the late Holocene. Quaternary Science Reviews. 28:1070-1083

DIRECCIÓN GENERAL DE AGUAS., 2008. Identificación de glaciares de roca. Volumen $\mathrm{N}^{\circ}$ 4. S.I.T $\mathrm{N}^{\circ} 167.31 \mathrm{p}$.

DIRECCIÓN GENERAL DE AGUAS., 2009. Inventario de glaciares descubiertos de las cuencas de los Ríos Elqui, Limarí Choapa. 18p.Inédito.

DEPARTAMENTO DE GEOFÍSICA, UNIVERSIDAD DE CHILE., 2006. Estudio de la variabilidad climática en Chile para el siglo XXI. Informe elaborado por el Departamento de Geofísica de la Universidad de Chile para CONAMA, 71.Inédito.

ERIKSON, I. y J. HÖGSTEDT., 2004. Landslide hazard assessment and landslide precipitation relationship in Valparaiso, central Chile. M. Sc. Thesis, Geography Department, Göteborg University, Göteborg. Inédito.
HAUSER. A., 1993. Remociones en masa en Chile. Boletín No 45. Servicio Nacional de Geología y Minería. Santiago, Chile. 75 pp.

HAUSER, A., 2002. Rock avalanche and resulting debris flow in Estero Parraguirre and Río Colorado, Región Metropolitana, Chile. In: Evans, S.G., DeGraff, J.V. (Eds.), Catastrophic Landslides; Effects, Occurrence, and Mechanisms. Reviews in Engineering Geology, vol. 15. Geological Society of America, pp. 135-148.

HARP, E.L. y R.C. WILSON., 1995. Shaking intensity thresholds for rock falls and slides: Evidence from the 1987 Whittier Narrows and Superstition Hills earthquake strong motion records. Bulletin of the Seismological Society of America, 85 (6): 1739-1757.

HUMLUM, O., 2000. The Geomorphic Significance of Rock Glaciers: estimates of rock glacier debris volumes and headwall recession rates in West Greenland. Geomorphology, 35: 41-67.

HEWITT, K., 2009. Rock avalanches that travel onto glaciers and related developments, Karakoram Himalaya, Inner Asia. Geomorphology, 103:66 -79.

IRIBARREN, P., 2008. Antecedentes de geomorfología paraglacial. Andes semiáridos de Chile. Anales de la Sociedad Chile de Ciencias Geográficas 2008: 76-81.

IRIBARREN, P. y X. BODIN., 2010. Geomorphic consequences of two large glacier and rock glacier destabilizations in the Central and Northern Chilean Andes. Geophysical Research Abstracts, EGU2010-7162-5, 2010.

IVERSON, R.M., LA HAUSEN, R.G., J.J ZIMMERMAN C.L., 1994. Debris flows against obstacles and bends: Dynamics and deposits. Procs., 1994 Fall Meeting, American Geophysical Union, p. 274. 
INTERNATIONAL SEISMOLOGICAL CENTER., 2009. On line Bulletin. Disponible en internet en: www.isc.ac.uk/ search/bulletin/

KÄÄB, A., C. HUGGEL, L. FISCHER, S. GUEX, F. PAUL, I. ROER, N. SALZMANN, S. SCHLAEFLI, K. SCHMUTZ, D. SCHNEIDER, T. STROZZI y W. WEIDMANN., 2005. Remote sensing of glacier- and permafrost-related hazards in high mountains: an overview. Natural Hazards and Earth System Sciences, 5: 527554.

KAWAGOE, S. S, KAZAMA. P, SARUKKALIGE., 2009. Assessment of snowmelt triggered landslide hazard and risk in Japan. Cold Regions Science and Technology. 1-10.

KRYSIECKI, J., BODIN, X., P, SCHOENEICH., 2008. Collapse of the Bérard Rock Glacier (Southern French Alps). Proceedings 9th International Conference on Permafrost, Fairbanks, Alaska, July 2008, pp. 153-154.

MATHEWSON, C.C., KEATON, J.R., SANTI, P.M., 1990. "Role of Bedrock Ground Water in the Initiation of Debris Flows and Sustained Post-Flow Stream Discharge," in The Bulletin of the Association of Engineering Geologists, Vol. 27, No. 1, pp. 73-83.

MOREIRAS, S.M., 2005. Climatic effect of ENSO associated with landslide occurrence in the Central Andes, Mendoza province, Argentina. Landslides, 2/1:53-59.

MPODOZIS, C. y P. CORNEJO., 1988. Hoja Pisco Elqui N ${ }^{\circ}$ 68 .Carta Geológica Nacional, IV Región de Coquimbo.1:250.000. SERNAGEOMÍN, Santiago.
NATIONAL OCEANIC AND ATMOSPHERIC ADMINISTRATION., 2009. ENSO Cycle: Recent Evolution, Current Status and Predictions. Disponible en Internet:http://www.cpc.noaa.gov/products/ analysis_monitoring/lanina/enso_evolution-status-fcsts-web.pdf

PARDO, M. MONFRET, T. VERA, T. EISENBERG, A.G, YAÑEZ., 2004. Crustal seismicity in the central Chile-western Argentina Andes cordillera. Geophysical Research Abstracts, Vol. 6, 04452.

PASKOFF, R., 1993. Geomorfología de Chile Semiárido. Universidad de La Serena, Fac de humanidades: 321p.La Serena.

SHULMEISTER ,J. T, DAVIES. D, EVANS. O, HYATT. D, TOVAR., 2009. Catastrophic landslides, glacier behaviour and moraine formation - A view from an active plate margin. Quaternary Science Reviews, 28: 1085-1096.

SCHROTT, L., 1996. Some geomorphological-hydrological characteristics of rock glaciers in the Andes, San Juan, Argentina. - In: Zeitschrift für Geomorphologie, Supplementband 104, 161-173.

VARNES, D. J., 1978. "Slope movements, types and processes", Landslides: Analysis and control, transportation rasearch board, Spec. Rep. 176, 11-33, National Academy of Science, Washington.

WILSON, R.C. y KEEFER, D.K., 1985. Predicting aerial limits of earthquake-induced landsliding. In, J.I. Ziony (ed.), Evaluating earthquake hazards in the Los Angeles region - An Earth-Science perspective, USGS Professional paper 1360, pp. 316-34. 